\title{
Motor variability - an important issue in occupational life
}

\author{
Divya Srinivasan* and Svend Erik Mathiassen \\ Centre for Musculoskeletal Research, Department of Occupational and Public Health Sciences, University of \\ Gavle, 80176 Gavle, Sweden
}

\begin{abstract}
Several recent reviews have reported that 'repetitive movements' is a risk factor for occupational musculoskeletal disorders (MSD) in the neck, shoulder and arm regions. More variation in biomechanical exposure is often suggested as an effective intervention in such settings. While increasing variation using extrinsic methods like job rotation may not always be possible in an industrial context, the intrinsic variability of the motor system may offer an alternative opportunity to increase variation. Motor variability (MV) refers to the natural variation in postures, movements and muscle activity observed to different extents in all tasks. The current review explores the state of the art in MV research from motor control, sports and occupational biomechanics literature to answer whether MV is important to consider in an occupational context, and if yes, whether this literature stimulates further studies to test if MV can be manipulated as a deliberate intervention for increasing biomechanical variation without jeopardizing production.
\end{abstract}

Keywords: Work-related musculo-skeletal disorders, exposure variability, motor variability, occupational health, performance, kinematics

\section{Introduction}

Working life is moving towards increased numbers of occupations characterized by long-lasting low levels of loads, maintaining static postures and/or performing repetitive actions (short-cycle assembly work, computerized office work, work in service sector). Musculoskeletal disorders (MSD) associated with repetitive work were identified as a significant problem in occupational life by researchers more than 20 years ago $[42,23]$, and the current trend in the nature of occupational work is making this issue of work-related MSD caused by repetitive work a critical and an urgent problem. The hand-arm, shoulder and low-back regions have been identified as those regions that are most susceptible to the occurrence of musculoskeletal disorders caused by performing repetitive/stereotypical work $[38,1]$.

Since repetitive work in the occupational context can be defined as long periods of 'identical' short- cycle operations being repeated over and over again [23], more 'variation in biomechanical exposure' (i.e. creating an exposure time-line that shows more changes in the temporal domain) is the most frequently suggested intervention against MSD caused by repetitive work. A number of epidemiological studies have suggested that jobs involving more physical variation most likely have a reduced risk for development of work-related MSD compared to jobs where similar movements are repeated over and over again (reviewed in [32]).

Accordingly, many ergonomic interventions focus on increasing exposure variation through 'extrinsic' methods such as: introducing new tasks into the job (as in job rotation scenarios); using different patterns of work and rest; making the work-task itself more variable, either by using different combinations of sub-tasks with varying performance requirements, or re-arranging the workstation. However, a number of such 'extrinsic' interventions have not yet received

\footnotetext{
* Corresponding author: Divya Srinivasan, Centre for Musculoskeletal Research, Department of Occupational and Public Health Sciences,
} University of Gavle, 80176 Gavle, Sweden.Ph: +46-026-64-8623. E-mail: divyasrinivasan@gmail.com 
firm empirical support for being effective [32]. Implementation of many such interventions may also not be feasible in all occupational scenarios from a production point of view.

Other ideas to increase exposure variation that have been explored are those that are 'intrinsic' to the task performer, where instead of focusing on changing something external in the work environment, the objective is to change the way the task is performed by the individual, i.e. his/her work technique. In this context, some newly emerging ideas to increase variation focus on the observation that there is a certain intrinsic variability natural to all motor control systems, whether in movement performance or posture control. Even under identical operating conditions, a movement is seldom repeated exactly the same way twice even if the person attempts to do so. So there is a certain cycle-to-cycle variability in kinematics (i.e. postures and movements) and muscle activation naturally present even in the most stereotypical tasks like a step or a reach, referred to as 'motor variability'. Although this concept of motor variability has existed for decades in motor control research, a new and potential solution to achieving more variation in occupational work may be to find ways to exploit this natural variation, while at the same time ensuring sufficient task performance.

This interest in motor variability and its potential to affect performance and health in occupational life is further justified in the light of recent studies which have shown that motor variability can be influenced by external working conditions - e.g. [12, 13, 4].

\section{What is Motor Variability and how can it be measured?}

Motor variability refers to the variability observed at different levels of movement execution, both within and between individuals: (a) in performanceoutcome measures like end-point precision, overall movement time or force developed; (b) in different kinetic or kinematic components of the movement pattern like joint angles/velocities, joint torques etc; (c) in muscle activity/recruitment patterns of muscles within or outside the same synergy, different regions within the same muscle or among multiple motor units making up each muscle region; and finally, (d) in 'coordinative' aspects of movements like relative joint angles/velocities, or variation in relative contribution of each muscle unit or joint towards achieving the final performance objective.
If motor variability is interpreted as sensorimotor noise, it is usually quantified by a cycle-to-cycle statistic of any chosen kinetic/kinematic variable/outcome-measure (standard deviation, coefficient of variation, inter-quartile range, median absolute deviation) or using geometric curve-based techniques like the ellipse method or centroid calculation [6]. However, increased applications of dynamic systems theories to movement control have led to the idea that motor variability is not just undesirable noise, but has a functional role in motor development and skill acquisition [3]. Such functional, chaotic variability has been investigated using several non-linear computational methods such as sample/approximate entropy, Lyapunov exponent etc $[45,46,13]$.

The amount of variability in an isolated movement component of a single body segment may not be sufficient to evaluate the information conveyed by MV, since most routine movements are complex coordinated movements involving multiple parts of the body/joints/muscles. So it is important to understand functional relationships between interacting movement components and their associated variabilities. Coordination variability can be assessed using variabilities of angle-angle plots, cyclograms, relative phase between joint angles, cross correlations and vector coding techniques (reviewed in [11]).

Based on the idea that motor variability arises from the redundant degrees of freedom available for performing multi-joint movements, concepts like the Uncontrolled Manifold Hypothesis (UCM - [40]) and Goal Equivalent Manifold Hypothesis (GEM - [10]) partition motor variability to a 'good' and 'bad' component, based on how the variability in movement components relates to variability in task performance. E.g. total motor variability in the joint configurations composing a movement is partitioned into (a) those patterns of motor variability that maintain the set of joint configurations such that the final task goal or control variable is satisfied, and are referred to as 'good' components of variability; (b) those patterns that result in a deviation from the final task goal or designated control variable (bad variability).

\section{What do we know about motor variability?}

This section reviews studies in occupational life (conducted in the field or using simulated occupational tasks in laboratory settings), as well as related studies in motor control, sports and 
rehabilitation which have assessed the relationship between motor variability and personal/individual factors such as pain, fatigue, performance and differences in individual traits/working techniques and experience. While the list of factors chosen or the list of studies within each section is by no means complete in terms of what has been done of relevance to motor variability in the different branches of research it touches upon, we have attempted to provide a comprehensive picture of the relationship between motor variability and basic variables of interest in an occupational health context.

\subsection{MV and individual traits}

From the perspective of work-related MSD in the neck-shoulder region, the need for a more thorough understanding of physiology and motor control strategies, in particular addressing individual differences, has been emphasized in several reviews on occupational disorders $[19,23,51]$. That individuals performing the same manual tasks differ significantly in their susceptibility to long-term chronic disorders has been attributed by some authors to differences in their motor patterns [24]. This notion is nourished by the general finding that individuals performing the same strictly controlled tasks differ considerably in their muscle coordination patterns $[20,35,50]$.

In a study of repetitive short-cycle assembly work in which subjects were required to repeatedly secure joints using hand-held nutrunners, a number of task factors such as types of joints, joint locations, types of tools etc were varied [34]. Significant interindividual differences were observed in variabilities of trapezius EMG and arm movement kinematics even if task factors were kept constant, and subjects reacted differently in terms of MV to changed task conditions. The authors report that these systematic differences between subjects in variability indicated individualized motor control strategies. Accordingly, physiological responses to activity, especially in the neck-shoulder region have also been found to differ substantially between subjects (e.g. [18, 21, 31, 47]).

An association between motor adaptation strategy and individual physiologic responses was suggested in another study on arm elevation in which subjects were required to maintain an isometric contraction for 15 minutes [33]. Individuals differed markedly in response, as reflected by EMG signals from different cranial and caudal compartments in the trapezius muscle, and by systemic responses. Three distinct subgroups of subjects were identified and these groups were suggested to represent different motor control scenarios, including differential engagement of subdivisions of the upper trapezius and alternating motor unit recruitment. The authors proposed that prolonged low-level contractions of the shoulder muscles involve neuromuscular adaptation strategies that may differ significantly between individuals.

In parallel to the growing interest in individual traits in motor strategies and their relevance to the occurrence of musculo-skeletal disorders in the occupational literature, researchers in sports sciences are also investigating a number of issues related to individual traits, especially from the perspective of task performance. A new tool known as coordination profiling [5] has been applied to examine how each individual uniquely satisfies task constraints during goaldirected behavior. Instead of the traditional approaches of pooling group data to study central tendencies and dispersions, this approach emphasizes on studying the unique ways in which individuals satisfy task constraints according to the intrinsic dynamics of their movement systems (reviewed in [11]).

Thus, individuals differ in motor strategies and the amount of motor variability exhibited while performing similar tasks. The next question is whether these differences have any association with performance and occupationally relevant physiological phenomena such as fatigue and pain.

\subsection{MV and pain}

Since pain is known to affect aspects of task performance, the relationship between pain and MV is potentially very interesting. In a comparison of the effects of experimental vs. chronic neck-shoulder pain on cycle-to-cycle variability of repetitive arm movements [29], the authors reported that pain alters the magnitude of motor variability: while experimental pain might be associated with increased variability since subjects try to explore alternate motor solutions to reduce pain, chronic pain might be characterized by decreased motor flexibility.

In a study of the patterns of motor variability associated with pain and experience in butchers performing a standardized cutting task[30], the authors observed that the motor variability of butchers increased during the first six months of employment, along with a corresponding decrease in the cycle-time variability. This was proposed to be a motor-learning effect. However, during these 6 months, half of the observed subjects developed neck-shoulder pain, and pain was found to decrease the variability in initial 
arm position, but increase the variability of the trunk. Kinematic motor variabilities of healthy, experienced butchers were higher when compared to the novice butchers. Since the healthy subjects had developed more variable motor strategies with experience, 'more variability in motor strategies' was proposed as a protective factor against the development of workrelated musculo-skeletal disorders.

In a subsequent butcher study conducted in the field [28], motor variability during a deboning process was found to vary with neck-shoulder discomfort, as well as experience. While longer work experience was associated with decrease in work cycle duration and decreased amount of variability in head-shoulder displacement (as reflected by linear estimates like cycle-to-cycle standard deviation and co-efficient of variation), the variability/complexity of the head-shoulder displacement using non-linear estimates like sample and approximate entropy showed a corresponding increase. Neck-shoulder discomfort in subjects was associated with lower motor variability (using both linear and non-linear estimation methods). Thus while there may be learning effects on motor variability, the authors concluded that pain/discomfort causes changes to motor variability through compensatory mechanisms.

In a study investigating performance of a repetitive reaching task until exhaustion in a healthy control group and a chronic neck-shoulder pain group [25], both groups were found to exhibit decreased relative arm variability with increasing fatigue. The pain group was suggested to have used a pain-minimizing strategy to accomplish the task that focused on increasing the contribution of non-painful joints (minimized shoulder movement and increased body center of mass movements), as against the control group which utilized more shoulder movements. In addition, the relative variability of the shoulder joint was increased in the pain group, suggesting that that people with shoulder pain may find it more difficult to control the movement of the shoulder joint.

Thus, pain may have a significant effect on MV. The mechanisms of short-term discomfort developing during task-performance or experimentally induced pain seem to be associated with an increase in motor variability, probably to find motor solutions that may reduce pain. On the other hand, longer term chronic pain seems to be associated with diminished motor variability/flexibility. This might be because in chronic pain/pathological conditions, the nervous system might prefer to use the most non-painful synergy (to avoid further pain) or in general because it may become more difficult to control painful joints
[25]. Hence, during chronic pain, motor solutions that use the painful joints in a more stereotypical manner or to a minimized extent may be preferred over other possible alternatives of performing the same task, even if they are less optimal from a performance standpoint [9].

\section{$3.3 \mathrm{MV}$ and Fatigue}

A number of studies on fatigue have shown that the muscular system has the capability to produce sustained force (perform isometric work) using variable spatio-temporal recruitment patterns, in order to increase endurance times: e.g. More spatio-temporal variability in EMG amplitude of muscles has been associated with longer endurance times to both sustained and intermittent isometric contractions of the corresponding muscles. E.g., even at low levels of activity in the trunk muscles, such as at $2-5 \% \mathrm{MVC}$, higher variability in EMG amplitude was shown to be associated with slower development of electromyographic manifestations of fatigue [48]. Similarly, more spatial variability of the EMG amplitude within the upper-trapezius muscle was correlated with the capacity to sustain shoulder contractions for a longer time [15]. People with more alternating activity between different parts of the erector spinae muscle had longer endurance times in isometric back extensions [49]; similarly it has been shown that load sharing between synergistic shoulder muscles with similar biomechanical functions might avoid or delay muscle fatigue development [39].

Fatigue and the spatial distribution of upper trapezius electromyographic (EMG) amplitude were compared during a 6-min constant force shoulder elevation task at $20 \%$ of the maximal voluntary contraction force (MVC) (constant force) and during the same task interrupted by brief, periodic increases in force to $25 \%$ MVC (variable force) [14]. During prolonged muscle activity, changes in spatial distribution of EMG over time reflected a mechanism to counteract fatigue, and periodic increases in force during a sustained contraction enhanced such modifications in the spatial distribution of upper trapezius EMG and further reduced fatigue, when compared to constant force contractions performed at a lower average load.

While the above studies discuss the effects of variable patterns of muscle recruitment on delaying the onset of fatigue or increasing endurance times in isometric work, studies have also reported similar effects in non-isometric, repetitive work. E.g. Withinsubject variability in repetitive isokinetic knee- 
extensions was inversely related to muscle fatigue, i.e., greater the variability in torque fluctuations implied that the muscle fatigued lesser during exercise [43]. Further, once fatigue sets in, increased variability in kinematic movement patterns have been observed - probably reflecting attempts to explore new motor solutions in order to relieve the load on a fatigued body region (similar to pain-adaptation strategies). E.g., a study of repetitive load lifting [44] reported decreased motion at the distal joints like the knee and hip being compensated by increased trunk motion.

\subsection{MV and performance}

In occupational settings, performance is critical from a production standpoint, and most often, the assessed from two main directions - (i) from the perspective of how a certain combination of objective, external work factors affect performance directly: E.g. how do changes in work-pace or lighting conditions or work-place design affect performance? (ii) How does performance change due to subjective factors such as fatigue, pain, work experience, psychosocial stress etc? These two directions are not entirely independent of each other, i.e., in most work-places, the external work factors interact with subjective factors such as fatigue/pain which would in turn affect performance. Motor variability may hold a key to this puzzle of interactions, since it may explain why with the same set of external work-factors, some people manage to stay healthy and unaffected, and can sustain good performance whereas others are affected, either from the perspective of health or performance or both.

A number of studies have reported that fatigue has adverse effects on performance, especially by increasing certain components of variability which are detrimental to the quality of performance: Precision of repetitive pointing movements was affected by muscle fatigue: variability of pointing (variable error) increased after fatigue due to decreased muscular cocontraction [36]. In a study on cross-country skiing [7], variation in angular displacement of arms and legs during skier's cyclic movements was described with a basic statistical measure of the magnitude of variation (standard deviation) and with nonlinear metrics for the structure of variation (Lyapunov exponent). The study showed a positive linear correlation between the degree of fatigue, variation magnitude (SD) and the degree of randomness (Lyapunov exponent) in the movement.
In an investigation of the effects of fatigue on kinematic variability during manual target tracking in repetitive cyclic movements [41], performance expressed as the time-on-target, was unaffected by fatigue. Since subjects changed their control strategy with increasing fatigue, although their overall kinematic variability increased, task performance was retained by staying closer to the center of the target when fatigued.

In complex multi-joint movements typical of routine day-to-day work tasks, the redundancy of the motor system makes it possible to alter motor patterns/strategies of movement organization in response to fatigue. Thus motor variability has been reported to play an important functional role in maintaining task performance, especially when performance is at a risk of deteriorating due to fatigue. E.g., subjects altered their movement patterns with fatigue, but in a way such that performance was not affected [17]. Similarly, expert table tennis players were found to be capable of maintaining task performance despite fatigue using such variable movement reorganization, whereas the performance of recreational players deteriorated with fatigue [2]. Evidence suggesting that changes in motor patterns may help in counteracting fatigue in order to preserve performance has also been reported in repetitive throwing $[16,22]$ and hammering tasks $[8,9]$.

Thus in section 3.3 , it was pointed out that MV could play a significant functional role in delaying the onset of fatigue, or in the evolution of compensatory mechanisms to relieve fatigue. The studies reviewed in section 3.4 indicate that MV may play an important functional role in maintaining optimal task performance despite the presence of fatigue, by movement reorganization/motor planning at the central organization level.

\subsection{MV and experience}

Long-term experience or 'skill development' in performing specific tasks or movements may imply that the motor system itself evolves more variable motor strategies. For example, when a set of healthy, experienced workers were compared with a reference group in performing standardized low-load repetitive work tasks [27], the experienced butchers exhibited motor strategies with more variable coordination patterns than the control group. The authors suggest that this increase in variability may be a possible protective strategy developed by the experienced workers which could, in turn, have been an important 
explanation for why they remained healthy. Similarly, in another study of standardized cutting tasks [30], kinematic motor variabilities of healthy, experienced butchers were higher when compared with novice butchers.

Some studies have also investigated the relationship between sports skills and motor variability, with practical implications for performance monitoring and training purposes. How coordination variability changes in relation with skills development in triple jump [52] showed that while beginners showed high coordination variability, skills training first decreased the amount of variability resulting in more consistent performance. However, in the final stages of skill development, variability increased again. The authors proposed that this was a functional variability that brought flexibility to the system, allowing it to cope with perturbations.

Thus contrary to traditional beliefs that experience decreases motor variability (on the assumption that motor variability was just undesirable motor noise), a number of studies have indicated that motor variability is important in order to make use of the redundancy built into the motor control system, and also to maintain movement stability and flexibility in order to cope with perturbations.

\section{Discussion}

Motor variability has been of great interest to neuroscience researchers for almost a century now, including its sources, effects and implications to neuromuscular control mechanisms. However, ways of utilizing this intrinsic variability in movement performance to obtain physiological benefits and the relevance of variable motor patterns/strategies to maintenance of the quality of task performance in occupational contexts have sprung up in both occupational and motor control literature only within the last decade.

This development has coincided with the recent agreement by both research and legislating bodies that disorders caused by repetitive work are a major issue in occupational life, and epidemiological evidence that increased exposure variation might be beneficial to musculo-skeletal health. Thus, within the last few years, a number of research efforts in the fields of ergonomics, physiology and motor control have started complementing one another to understand the relevance and possible contributions of motor variability to health in working life. Among these, some notable efforts have been made to understand motor variability and its effects with a focus on occupational life include work [32, 29, 30, 28, 26, 52, 12, $8,9,4]$. Because of its relevance to both physiological responses as well as performance, this growing interest in motor variability is also shared by clinicians [37], and researchers in sports biomechanics [3], the latter emphasizing issues similar to the ones raised in the present paper concerning performance and learning.

Since more variable movements have been associated with later onset of fatigue and also with increased performance quality in some cases [8, 17], it is desirable to set the different work-factors that could affect movements in an occupational environment such that less stereotypical movements are encouraged, but simultaneously taking care to ensure the quality/quantity of production is not negatively affected. The effects of some external work factors such as work-pace [4], or personal factors such as subjective pain and skills [29, 30] and fatigue [25] on certain aspects of movement variability like task timing have been studied in the past with promising results.

However, there has been no concerted effort until now to systematically analyze the effects of several factors commonly occurring in occupational settings, and their possible interactions, on the different spatiotemporal aspects of movement variability - e.g. work pace, precision requirements, spatial and temporal autonomy in task performance, and added mental load. The relationships between variable motor patterns, performance and physiological responses such as pain and fatigue, and how these complex relationships change with time, in prolonged, repetitive, short-cycle assembly tasks will help in answering questions that are quite novel in occupational research: e.g. whether changes in motor patterns occur as an anticipatory physiological response to delay the onset of fatigue/pain, or whether they occur in reaction to the occurrence of physiological indicators like pain or fatigue, to ensure there is no loss in the quality of production.

A number of these research issues have not so far been investigated properly because of the absence of a standardized method(s) to quantify motor variability. Motor control research has developed several sophisticated methods to analyze motor variability (e.g. for partitioning motor variability into certain 'good' and 'bad' components). However, these methods have only been applied to simple and controlled experiments conducted in lab settings, and have not been applied in the field, and not to (simulated) occu- 
pational tasks. Hence, those studies on repetitive work tasks in the ergonomics literature that deal with MV use very primitive variables for measuring it [32]. Bridging this disconnect between research methods available in the motor control literature and methods currently applied in occupational research can help in the development of simple, operational methods to quantify motor variability, applicable in field studies.

\section{Conclusion}

Thus, after a systematic review of the existing literature on MV in the fields of motor control, sports and occupational biomechanics, it seems justified to conclude that motor variability is a viable means of increasing biomechanical variation in occupational work without jeopardizing production. A unified approach to quantify variation in occupational settings, what aspects of movement variation are relevant to working life in terms of their effects on health and production, whether a certain optimal combination of work factors can produce desired changes in motor variability, and finally, how suitable ergonomic interventions can be designed such that alternative motor solutions and recovery are promoted, without compromising on performance are all questions very relevant to occupational health and performance.

\section{References}

[1] Arbetsorsakade besvär 2010: www.av.se/statistik/ officiellt/arbetsorsakade besvar 2010.aspx

[2] Aune, T. K., R. P. Ingvaldsen, et al. (2008). "Effect of physical fatigue on motor control at different skill levels." Percept Mot Skills 106(2): 371-86.

[3] Bartlett, R., J. Wheat, et al. (2007). "Is movement variability important for sports biomechanists?" Sports Biomech 6(2): 224-43.

[4] Bosch, T., S. E. Mathiassen, et al. (2011). "The effect of work pace on workload, motor variability and fatigue during simulated light assembly work." Ergonomics 54(2): 154-168.

[5] Button, C. and K. Davids (1999). "Interacting intrinsic dynamics and intentionality requires coordination profiling of movement systems." Studies in Perception and Action V 5: 314-318.

[6] Chau, T., S. Young, et al. (2005). "Managing variability in the summary and comparison of gait data." J Neuroeng Rehabil 2: 22.

[7] Cignetti, F., F. Schena, et al. (2009). "Effects of fatigue on inter-cycle variability in cross-country skiing." Journal of Biomechanics 42(10): 1452-1459.

[8] Cote, J. N., A. G. Feldman, et al. (2008). "Effects of fatigue on intermuscular coordination during repetitive hammering." Motor Control 12(2): 79-92.

[9] Cote, J. N., D. Raymond, et al. (2005). "Differences in multi-joint kinematic patterns of repetitive hammering in healthy, fatigued and shoulder-injured individuals." Clinical Biomechanics 20(6): 581-590.

[10] Cusumano, J. P. and P. Cesari (2006). "Body-goal variability mapping in an aiming task." Biol Cybern 94(5): 367-79.

[11] Davids, K., P. Glazier, et al. (2003). "Movement systems as dynamical systems: the functional role of variability and its implications for sports medicine." Sports Med 33(4): 245-60.

[12] Dempsey, P. G., S. E. Mathiassen, et al. (2010). "Influence of three principles of pacing on the temporal organisation of work during cyclic assembly and disassembly tasks." Ergonomics 53(11): 1347-1358.

[13] Dingwell, J. B. and L. C. Marin (2006). "Kinematic variability and local dynamic stability of upper body motions when walking at different speeds." Journal of Biomechanics 39(3): 444-452.

[14] Falla, D. and D. Farina (2007). "Periodic increases in force during sustained contraction reduce fatigue and facilitate spatial redistribution of trapezius muscle activity." Experimental Brain Research 182(1): 99-107.

[15] Farina, D., F. Leclerc, et al. (2008). "The change in spatial distribution of upper trapezius muscle activity is correlated to contraction duration." J Electromyogr Kinesiol 18(1): 16-25.

[16] Forestier, N. and V. Nougier (1998). "The effects of muscular fatigue on the coordination of a multijoint movement in human." Neuroscience Letters 252(3): 187-190.

[17] Gates, D. H. and J. B. Dingwell (2008). "The effects of neuromuscular fatigue on task performance during repetitive goal-directed movements." Exp Brain Res 187(4): 573-85

[18] Hagberg, M. (1981). "Work load and fatigue in repetitive arm elevations." Ergonomics 24(7): 543-55.

[19] Hagberg, M. (1992). "Exposure variables in ergonomic epidemiology." Am J Ind Med 21(1): 91-100.

[20] Hammarskjold, E., K. Harmsringdahl, et al. (1990). "Shoulder Arm Muscular-Activity and Reproducibility in Carpenters Work." Clinical Biomechanics 5(2): 8187.

[21] Hansson, G. A., U. Stromberg, et al. (1992). "Electromyographic fatigue in neck/shoulder muscles and endurance in women with repetitive work." Ergonomics 35(11): 1341-52.

[22] Huffenus, A. F., N. Forestier, et al. (2006). "Effects of distal and proximal arm muscles fatigue on multi-joint movement organization." Experimental Brain Research 170(4): 438-447.

[23] Kilbom, A. (1994). "Assessment of physical exposure in relation to work-related musculoskeletal disorders-what information can be obtained from systematic observations?" Scand J Work Environ Health 20 Spec No: 30-45.

[24] Kilbom, A. and J. Persson (1987). "Work technique and its consequences for musculoskeletal disorders." Ergonomics 30(2): 273-9.

[25] Lomond, K. V. and J. N. Cote (2010). "Movement timing and reach to reach variability during a repetitive reaching task in persons with chronic neck/shoulder pain and healthy subjects." Experimental Brain Research 206(3): 271-282

[26] Madeleine, P. (2010). "On functional motor adaptations: from the quantification of motor strategies to the prevention of musculoskeletal disorders in the neck- 
shoulder region (vol 199, pg 1, 2010)." Acta Physiologica 200(3): 289-289.

[27] Madeleine, P., B. Lundager, et al. (2003). "Standardized low-load repetitive work: evidence of different motor control strategies between experienced workers and a reference group." Applied Ergonomics 34(6): 533-542.

[28] Madeleine, P. and T. M. T. Madsen (2009). "Changes in the amount and structure of motor variability during a deboning process are associated with work experience and neck-shoulder discomfort." Applied Ergonomics 40(5): 887-894

[29] Madeleine, P., S. E. Mathiassen, et al. (2008a). "Changes in the degree of motor variability associated with experimental and chronic neck-shoulder pain during a standardised repetitive arm movement." Experimental Brain Research 185(4): 689-698

[30] Madeleine, P., M. Voigt, et al. (2008b). "The size of cycle-to-cycle variability in biomechanical exposure among butchers performing a standardised cutting task." Ergonomics 51(7): 1078-1095.

[31] Mathiassen, S. E. (1993). "The Influence of Exercise/Rest Schedule on the Physiological and Psychophysical Response to Isometric Shoulder-Neck Exercise." European Journal of Applied Physiology and Occupational Physiology 67(6): 528-539.

[32] Mathiassen, S. E. (2006). "Diversity and variation in biomechanical exposure: What is it, and why would we like to know?" Applied Ergonomics 37(4): 419-427.

[33] Mathiassen, S. E. and T. Aminoff (1997). "Motor control and cardiovascular responses during isoelectric contractions of the upper trapezius muscle: evidence for individual adaptation strategies." European Journal of Applied Physiology 76(5): 434-444.

[34] Mathiassen, S. E., T. Moller, et al. (2003). "Variability in mechanical exposure within and between individuals performing a highly constrained industrial work task." Ergonomics 46(8): 800-824.

[35] Mathiassen, S. E. and J. Winkel (1996). "Physiological comparison of three interventions in light assembly work: Reduced work pace, increased break allowance and shortened working days." International Archives of Occupational and Environmental Health 68(2): 94-108.

[36] Missenard, O., D. Mottet, et al. (2008). "The role of cocontraction in the impairment of movement accuracy with fatigue." Experimental Brain Research 185(1): 151-156.

[37] Moseley, G. L. and P. W. Hodges (2006). "Reduced variability of postural strategy prevents normalization of motor changes induced by back pain: A risk factor for chronic trouble?" Behavioral Neuroscience 120(2): 474-476.

[38] National Research Council (2001). Musculoskeletal Disorders and the Workplace. Washington DC: National Academies Press.

[39] Palmerud, G., H. Sporrong, et al. (1998). "Consequences of trapezius relaxation on the distribution of shoulder muscle forces: an electromyographic study." Journal of Electromyography and Kinesiology 8(3): 185-193.

[40] Schoner, G. (1995). "Recent developments and problems in human movement science and their conceptua implications." Ecological Psychology 7(4): 291-314.

[41] Selen, L. P. J., P. J. Beek, et al. (2007). "Fatigueinduced changes of impedance and performance in target tracking." Experimental Brain Research 181(1): 99108
[42] Silverstein, B., L. Fine, et al. (1987). "Hand-wrist disorders among investment casting plant workers." J Hand Surg Am 12(5 Pt 2): 838-44.

[43] Skurvydas, A., M. Brazaitis, et al. (2010). "Prolonged Muscle Damage Depends on Force Variability." International Journal of Sports Medicine 31(2): 77-81.

[44] Sparto, P. J., M. Parnianpour, et al. (1997). "The effect of fatigue on multijoint kinematics and load sharing during a repetitive lifting test." Spine 22(22): 2647 2654.

[45] Stergiou, N., R. Harbourne, et al. (2006). "Optimal movement variability: a new theoretical perspective for neurologic physical therapy." J Neurol Phys Ther 30(3): 120-9.

[46] Stergiou, N., C. Moraiti, et al. (2004). "The effect of the walking speed on the stability of the anterior cruciate ligament deficient knee." Clinical Biomechanics 19(9): 957-963.

[47] Sundelin, G. (1993). "Patterns of electromyographic shoulder muscle fatigue during MTM-paced repetitive arm work with and without pauses." Int Arch Occup Environ Health 64(7): 485-93.

[48] van Dieen, J. H., E. P. Westebring-van der Putten, et al. (2009). "Low-level activity of the trunk extensor muscles causes electromyographic manifestations of fatigue in absence of decreased oxygenation." Journal of Electromyography and Kinesiology 19(3): 398-406.

[49] van Dieen, J. H., H. H. E. O. Vrielink, et al. (1993). "An Investigation into the Relevance of the Pattern of Temporal Activation with Respect to Erector Spinae Muscle Endurance." European Journal of Applied Physiology and Occupational Physiology 66(1): 70-75.

[50] Westgaard, R. H., C. Jensen, et al. (1993). "Individual and work-related risk factors associated with symptoms of musculoskeletal complaints." Int Arch Occup Environ Health 64(6): 405-13.

[51] Westgaard, R. H. and J. Winkel (1996). "Guidelines for occupational musculoskeletal load as a basis for intervention: a critical review." Appl Ergon 27(2): 79-88.

[52] Wilson, C., S. E. Simpson, et al. (2008). "Coordination variability and skill development in expert triple jumpers." Sports Biomechanics 7(1): 2-9. 